\title{
ON BEST CONDITIONED MATRICES ${ }^{1}$
}

G. E. FORSYTHE AND E. G. STRAUS

1. Main theorems. Let $A$ be a positive definite Hermitian matrix of finite order, and let $\Lambda$ and $\lambda$ be its maximal and minimal eigenvalue respectively. The condition number of $A$ is the ratio $P(A)=\Lambda / \lambda$ introduced by Todd [1]. Let $\mathcal{T}$ be a class of regular linear transformations. Define $A^{T}=T^{*} A T$. We say that $A$ is best conditioned with respect to $\mathcal{T}$ if $P\left(A^{T}\right) \geqq P(A)$ for all $T \in \mathcal{T}$.

In order to investigate whether $A$ is best conditioned we remember that

$$
\Lambda=\max _{x} \frac{x^{*} A x}{x^{*} x}, \quad \lambda=\min _{x} \frac{x^{*} A x}{x^{*} x}
$$

and hence

$$
P(A)=\max _{\|x\|=\|v\|=1} \frac{x^{*} A x}{y^{*} A y} .
$$

We introduce the abbreviation $R=R(T)=\left(T^{*}\right)^{-1} T^{-1}$. Now let $\Lambda^{T}, \lambda^{T}$ be the extremal eigenvalues of $A^{T}$. Setting $u=T x$, we obtain from (1) and (2):

$$
\begin{aligned}
\Lambda^{T} & =\max _{x} \frac{x^{*} T^{*} A T x}{x^{*} x}=\max _{u} \frac{u^{*} A u}{u^{*} R u}, \\
\lambda^{T} & =\min _{x} \frac{x^{*} T^{*} A T x}{x^{*} x}=\min _{u} \frac{u^{*} A u}{u^{*} R u}, \\
P\left(A^{T}\right) & =\max _{\|u\|=\|v\|=1} \frac{u^{*} A u}{v^{*} A v} \cdot \frac{v^{*} R v}{u^{*} R u} .
\end{aligned}
$$

Thus, if we let $S_{\Lambda}, S_{\lambda}$ be the sets of unit eigenvectors of $A$ belonging to $\Lambda$ and $\lambda$ respectively, then we obtain from (2)

$$
P(A)=\frac{x^{*} A x}{y^{*} A y}, \quad x \in S_{\Lambda}, y \in S_{\lambda} .
$$

Presented to the International Congress of Mathematicians, September 8, 1954; received by the editors August 13, 1954 .

1 The first author wishes to acknowledge the sponsorship of the Office of Naval Research-at the beginning of this research through the National Bureau of Standards, Los Angeles, and now in connection with Project ONR 044-144. The authors wish to acknowledge helpful suggestions by their colleague Dr. T. S. Motzkin. 
Hence from (3) and (4)

$$
P\left(A^{T}\right) \geqq P(A) \max _{u \in S_{\Lambda}, v \in S_{\lambda}} \frac{v^{*} R v}{u^{*} R u} .
$$

We thus have proved:

Lemma. If $\max _{u \in S_{\Lambda}, v \in S_{\lambda}}\left(v^{*} R v / u^{*} R u\right) \geqq 1$ for all $T \in \mathcal{T}$, then $A$ is best conditioned with respect to $\mathcal{T}$.

It will be convenient to introduce the concept of "separability by $\tau^{\prime \prime}:$

Definition. The sets $S_{1}, S_{2}$ are separable by $\mathcal{\sigma}$ if there exists a $T \in \mathcal{T}$ and a constant $k$ so that

$$
x^{*} R x<k<y^{*} R y,
$$

for all $x$ in one $S_{j}$ and all $y$ in the other.

Obviously, if $S_{1}, S_{2}$ are not separable by $\mathcal{T}$, then

$$
\sup _{x \in S_{1}, y \in S_{2}} \frac{x^{*} R x}{y^{*} R y} \geqq 1 \quad \text { for all } T \in \mathcal{T} .
$$

Combining (5) with the lemma, we have proved

Theorem 1. If. $S_{\Lambda}$ and $S_{\lambda}$ are not separable by $\mathcal{T}$, then $A$ is best conditioned with respect to $\mathcal{T}$.

The converse to Theorem 1 is not true without further conditions on $\mathcal{T}$. As such a condition we introduce the following concept:

Definition. A set $\mathcal{T}$ of regular linear transformations is called infiniiesimally complete if, for every $T \in \mathcal{T}$, there exist arbitrarily small positive $\epsilon, \epsilon^{\prime}$ such that there are $T_{\epsilon}, T_{\epsilon^{\prime}} \in \mathcal{T}$ with

$$
I+\epsilon R=c\left(T_{\epsilon}^{*}\right)^{-1} T_{\epsilon}^{-1}, \quad I-\epsilon^{\prime} R=c^{\prime}\left(T_{\epsilon^{\prime}}^{*}\right)^{-1} T_{\epsilon^{\prime}}^{-1},
$$

where $c, c^{\prime}$ are (positive) numbers.

THEOREM 2. If $\mathcal{T}$ is infinitesimally complete and $S_{\lambda}, S_{\Lambda}$ are separable by $\mathcal{T}$, then $A$ is not best conditioned with respect to $\mathcal{T}$.

Proof. By the hypothesis of separability there exists a $T \in \mathcal{T}$ and a $k>0$ such that either

$$
x^{*} R x>k>y^{*} R y \quad \text { for all } x \in S_{\Lambda}, y \in S_{\lambda},
$$

or

$$
x^{*} R x<k<y^{*} R y \quad \text { for all } x \in S_{\Lambda}, y \in S_{\lambda} .
$$


In case (I) we have $y^{*} R y / x^{*} R x<1$ for all $x \in S_{\Lambda}, y \in S_{\lambda}$. Hence there exist neighborhoods $U_{\Lambda}, U_{\lambda}$ of $S_{\Lambda}, S_{\lambda}$ on the unit sphere $S$ so that for every $\epsilon>0$

$$
\sup _{x \in U_{\Lambda}, \nu \in U_{\lambda}} \frac{y^{*}(I+\epsilon R) y}{x^{*}(I+\epsilon R) x}<1 .
$$

Define $U$ to be the Cartesian product $U_{\Lambda} \times U_{\lambda}$. Then

$$
\left(\max _{(x, y) \in U} \frac{x^{*} A x}{y^{*} A y}\right) \cdot\left(\sup _{(x, y) \in U} \frac{y^{*}(I+\epsilon R) y}{x^{*}(I+\epsilon R) x}\right)<P(A) .
$$

Let $F=S \times S-U$. Then $\max _{(x, y) \in F}\left(x^{*} A x / y^{*} A y\right)<P(A)$. Hence we may fix $\epsilon$ so small that

$$
\left(\max _{(x, y) \in_{F}} \frac{x^{*} A x}{y^{*} A y}\right) \cdot\left(\max _{(x, y) \in_{F}} \frac{y^{*}(I+\epsilon R) y}{x^{*}(I+\epsilon R) x}\right)<P(A) .
$$

By the infinitesimal completeness of $\mathcal{T}$, there is a $T_{\epsilon} \in \mathcal{T}$ such that

$$
\frac{y^{*} R_{\epsilon} y}{x^{*} R_{\epsilon} x}=\frac{y^{*}(I+\epsilon R) y}{x^{*}(I+\epsilon R) x}, \quad \text { where } R_{\epsilon}=R\left(T_{\epsilon}\right) .
$$

Putting (8) into (6) and (7), we then see from (3) that

$$
P\left(A^{T_{\epsilon}}\right)<P(A) \text {. }
$$

The proof of (9) in Case (II) is entirely analogous, if we replace $I+\epsilon R$ in (6), (7), (8) by $I-\epsilon^{\prime} R$. But (9) proves the theorem.

2. Applications. As examples of infinitesimally complete classes $\mathcal{T}$ we may cite:

(i) Quasidiagonal matrices. These are matrices of form

$$
T=\left[\begin{array}{cccc}
M_{1} & 0 & \cdots & 0 \\
0 & M_{2} & \cdots & 0 \\
: & \vdots & & \vdots \\
\dot{0} & 0 & \cdots & M_{8}
\end{array}\right],
$$

where each $M_{i}$ is a square matrix of arbitrary preassigned order. An important subclass is the following:

(ii) Diagonal (or real diagonal or positive diagonal) matrices $D$. Here forming $D^{*} A D$ is a special case of the common practice of preconditioning $A$ by scaling rows and columns. This is used, for example, to make $A$ more easily invertible by a numerical process. For numerical operations on a general nonsingular matrix $C$ the condition 
number $P(A)$, where $A=C C^{*}$, is often significant. Preconditioning of $C$ by scaling rows alone yields a matrix $C_{1}=D^{*} C$, for which $C_{1} C_{1}^{*}$ $=D^{*} A D$. Minimizing $P\left(D^{*} A D\right)$ (at least approximately) thus has practical importance for both Hermitian and general matrices.

If $D$ is a regular diagonal matrix, then $x^{*} R x$ assumes the particularly simple form

$$
x^{*} R x=x^{*}\left(D^{*}\right)^{-1} D^{-1} x=\sum_{i=1}^{n}\left|d_{i i}\right|^{-2}\left|x_{i}\right|^{2} .
$$

Thus separability by class (ii) means $S_{\Lambda}$ and $S_{\lambda}$ can be separated by an axis-oriented, origin-centered ellipsoid. From (10) we can establish

Theorem 3. $A$ sufficient condition for $A$ to be best conditioned with respect to class (ii) is that, for some pair of eigenvectors $x^{\Lambda}, x^{\lambda}$ belonging to $\Lambda, \lambda$,

$$
\left|x_{i}^{\Lambda}\right|=\left|x_{i}^{\lambda}\right| \quad(i=1, \cdots, n) .
$$

Moreover, if $\Lambda, \lambda$ are simple eigenvalues, (11) is also necessary.

Proof. If (11) holds, then, by (10), $x^{*} R x$ assumes the same value for both $x^{\Lambda}$ and $x^{\lambda}$. The sufficiency then follows from Theorem 1. On the other hand, if $\Lambda$ and $\lambda$ are simple then $S_{\Lambda}, S_{\lambda}$ consist of two points each. We then see that (11) is necessary and sufficient for separability of $S_{\Lambda}$ and $S_{\lambda}$. This proves the necessity.

Note that (11) says that $x^{\Lambda}, x^{\lambda}$ are reflections of each other in some coordinate subspace.

When $\Lambda$ or $\lambda$ is multiple, there are inseparable $S_{\Lambda}, S_{\lambda}$ containing no $x^{\Lambda}, x^{\lambda}$ which are reflections of each other. For an example of this, let

$$
A=\left[\begin{array}{ccc}
1 & \alpha & \alpha \\
\alpha & 1 & \alpha \\
\alpha & \alpha & 1
\end{array}\right], \quad 0<\alpha<1
$$

Here $\Lambda=1+2 \alpha ; \quad S_{\Lambda}$ consists of two points $\pm P$, where $P$ $=(1,1,1) / 3^{1 / 2}$. Also $\lambda=1-\alpha$ (double root), and $S_{\lambda}$ is the circle $x+y+z=0, x^{2}+y^{2}+z^{2}=1$. Now we show that it is impossible to separate $S_{\lambda}$ from $S_{\Lambda}$ by any quadratic surface $f(x, y, z)=a x^{2}+b y^{2}$ $+c z^{2}=d$. First, $f(P)=(a+b+c) / 3$. Let $r=1 / 2^{1 / 2}$. Take, on $S_{\lambda}, P_{1}$ $=(r,-r, 0), P_{2}=(r, 0,-r)$, and $P_{3}=(0, r,-r)$. Then $f\left(P_{1}\right)$ $=(a+b) / 2, f\left(P_{2}\right)=(a+c) / 2$ and $f\left(P_{3}\right)=(b+c) / 2$. Hence $f(P)=\left[f\left(P_{1}\right)\right.$ $\left.+f\left(P_{2}\right)+f\left(P_{3}\right)\right] / 3$, and $f(P)$ must lie between the extreme values of the $f\left(P_{i}\right)$. 
Theorem 3 will be applied to prove a conjecture of Young [2]. The conjecture is significant for an iterative solution of certain systems of linear equations.

Theorem 4. A positive definite Hermitian matrix of form

$$
Q=\left[\begin{array}{ll}
I_{p} & B \\
B^{*} & I_{q}
\end{array}\right]
$$

where $I_{p}, I_{q}$ are unit matrices, is always best conditioned with respect to class (ii).

Proof. Let $r$ be the rank of $B$. The semidefinite matrix $B^{*} B$ has exactly $r$ positive eigenvalues $\nu_{i}^{2}$, which we number so that $0<\nu_{1}^{2}$ $\leqq \cdots \leqq \nu_{r}^{2}$. Let $B^{*} B y_{i}=\nu_{i}^{2} y_{i}$. One finds that the partitioned vectors $\left(B y_{i}, \pm \nu_{i} y_{i}\right)$ are $2 r$ linearly independent eigenvectors of $Q$ belonging to the $2 r$ eigenvalues $1 \pm \nu_{i}(i=1, \cdots, r)$. Since $Q$ is definite, all $\nu_{i}<1$.

If $p-r>0$, there are $p-r$ linearly independent vectors $u_{j}$ with $B^{*} u_{j}=0$. Then $\left(u_{j}, 0\right)$ are $p-r$ linearly independent eigenvectors of $Q$ belonging to the eigenvalue 1 . Similarly, if $q-r>0$, there are $q-r$ linearly independent eigenvectors of $Q$ of type $\left(0, v_{k}\right)$, which all belong to the eigenvalue 1 . Here all $B v_{k}=0$.

We have found all $p+q$ eigenvalues of $Q$, and see that the largest is $\Lambda=1+\nu_{r}$, with an eigenvector $\left(B y_{r}, \nu_{r} y_{r}\right)$. The smallest is $\lambda=1-\nu_{r}$ with an eigenvector $\left(B y_{r},-\nu_{r} y_{r}\right)$. Theorem 3 then completes the proof.

For any scalar $c, P\left(c D^{*} Q D\right)=P\left(D^{*} Q D\right)$. It would be interesting to know, for the $Q$ of (12), when the class $c Q$ contains all the best conditioned transforms $D^{*} Q D$. These transforms essentially constitute the matrices with Young's Property A [3], often encountered in the numerical solution of partial differential equations.

We can show that the partitioned positive definite matrices

$$
\left[\begin{array}{cc}
C_{1} I_{p} & B \\
B^{*} & c_{2} I_{q}
\end{array}\right]
$$

are best conditioned if and only if $c_{1}=c_{2}$. On the other hand, the third order matrices

$$
Q=\left[\begin{array}{lll}
1 & 0 & b \\
0 & d & 0 \\
b & 0 & 1
\end{array}\right]
$$


where $|b|<1$ and $1-|b| \leqq d \leqq 1+|b|$, are all best conditioned, with $P(Q)=(1+|b|) \cdot(1-|b|)^{-1}$. We conjecture that, for $Q$ as in (12), any best conditioned matrix $D^{*} Q D \neq c Q$ has the form

$$
\Pi^{*}\left[\begin{array}{ccc}
c I_{p_{1}} & 0 & B_{1} \\
0 & D_{p_{2}} & 0 \\
B_{1}^{*} & 0 & c I_{q}
\end{array}\right] \Pi
$$

where $D_{p_{2}}$ is diagonal and $\Pi$ is a permutation matrix.

\section{REFERENCES}

1. John Todd, The condition of a certain matrix, Proc. Cambridge Philos. Soc. vol. 46 (1950) pp. 116-118.

2. David Young, On the solution of linear systems by iteration, Proceedings of the Sixth Symposium in Applied Mathematics, to be published.

3. - Iterative methods for solving partial difference equations of elliptic type, Trans. Amer. Math. Soc. vol. 76 (1954) pp. 92-111.

University of California, Los Angeles 